\title{
Folk name and lore of birds from the Sundanese of West Java, Indonesia: An ethno-ornithological survey
}

\author{
DEDE MULYANTO ${ }^{1}$, JOHAN ISKANDAR ${ }^{2}$, ALIYA MADANI ${ }^{1}$, RIMBO GUNAWAN $^{1}$, \\ RUHYAT PARTASASMITA ${ }^{2, \bullet}$ \\ ${ }^{1}$ Department of Anthropology, Faculty of Social and Political Sciences, Universitas Padjadjaran. Jl. Raya Bandung-Sumedang Km 21 Jatinangor, \\ Sumedang 45363, West Java, Indonesia \\ ${ }^{2}$ Department of Biology, Faculty of Mathematics and Natural Sciences, Universitas Padjajaran. Jl. Raya Bandung-Sumedang Km 21, Jatinangor, \\ Sumedang 45363, West Java, Indonesia. Tel.: +62- 22-7797712. `email: ruhyat.partasasmita@unpad.ac.id, rp2010rikkyo@gmail.com
}

Manuscript received: 1 July 2020. Revision accepted: 29 August 2020.

\begin{abstract}
Mulyanto D, Iskandar J, Madani A, Gunawan R, Partasasmita R. 2020. Folk name and lore of birds from the Sundanese of West Java, Indonesia: An ethno-ornithological survey. Biodiversitas 21: 4384-4395. Since last time, research on birds in West Java had been undertaken by ornithologists. The ethnoornithology research, however, has been rarely employed. The study aims were to account for vernacular or folk names, folk classification, and folkloric birds based on case of the mountain people of Keratasari, West Java, Indonesia. The method in this study was qualitative with the ethnoornithological approach. The field data were collected by focus group discussion and deep interviews with informants of 12 groups of independent village people of four villages of Kertasari sub-district. The naming of collected data was by systematic elicitation of names from pictorial representations of birds and organized here to facilitate analysis of various aspects of folk taxonomy about the scientific one. Folklore about birds that were collected in natural contexts is also included to indicate the birds' role and their names in symbolic processes that exceed the limits of literal reference. The result of the study showed that it was recorded 222 bird species, representing 170 vernacular names, 93 of them were recorded by Koningsberger (1901-1909). The taxonomic and folkloric mode of knowledge in this paper presents that birds play important roles in villagers' lives of Sundanese people, particularly in the study area. Generally, birds have been an important role in socio-cultural aspects, including in folklore of Sundanese people who reside in rural mountain areas of West Java.
\end{abstract}

Keywords: Folk classification, folkloric birds, folk name, Sundanese, West Java

\section{INTRODUCTION}

Based on ecological history, the Sundanese people of West Java were highland dwellers whose livelihoods were mainly based on shifting cultivation and forest products (Nastiti 2006), and only after the end of the $18^{\text {th }}$ century, some of them began to settle in the lower areas and became sawah cultivators (Geertz 1963; Iskandar and Iskandar 2011). The process of 'sawahisation', however, did not occur equally in all-region. Until the beginning of the $19^{\text {th }}$ century, the mountainous region of West Java was still inhabited by shifting cultivators, and only at the end of the century, when the expansion of modern plantations reached its peak, this lifestyle finally disappeared from this region (Svensson 1991; Breman 2015). Except for relict rainforest surviving area, mostly in the upland southwest Java, the traditional shifting cultivation system has strongly been maintained by traditional Sundanese people of Baduy, South Banten, and Kasepuhan community of Cisolok, Sukabumi (Iskandar 2012).

Since the Sundanese rural people of mountainous region used to practice shifting cultivation, they own close relationships with the environment. Indeed, they have rich local knowledge on various local biological components, including birds. The local knowledge (LK) or the traditional ecological knowledge (TEK) can be defined as "a cumulative body of knowledge, practice, and belief, evolving by adaptive process and handed down through generations by cultural transmission, about the relationship of a living being (including humans) with one another and with their environment" (Berkes 2008). Birds have occupied special place in the lives of the rural Sundanese people of West Java (Iskandar. Avifauna has been an important role in ecological and socio-economic and cultural functions of rural people (Iskandar 2017). The rural Sundanese people, like other traditional people around the world, have served the purpose of birds as climate and weather prognosticator, agricultural augury, pet, meat, traditional medicine, ritual, myth, and symbolism (Dove 1993; Forth 2000; Alves 2012; Agnihotri and Si 2012; Bezerra et al.2013; Roldlan-Clara et al. 2014; Teixeira el al. 2014; Deikumah et al. 2015; Kane 2015; Iskandar et al. 2016; Iskandar 2017; Pam 2017; Hull and Fergus 2017; Pam 2017).

In the past, some studies on birds in West Java were undertaken by Dutch ornithologists, including Koningsberger $(1901,1909)$ and Hoogerwerf (1948; $\left.1949^{\mathrm{a}}, 1949^{\mathrm{b}}\right)$. Based on this study, it was recorded various bird species with vernacular names, particularly based on Sundanese language. For example, based on Koningsberger who collected directly from the native people in the surrounding valley of Mount Gede-Pangrango complex, the area of about $70 \mathrm{~km}$ westward of West Java, it was 
documented at least 113 vernacular bird names based on Sundanese language.

Nowadays, birds of rural mountain ecosystems of West Java have dramatically decreased due to many factors, including forest conversion to other land uses, illegal hunting of birds, and intensive pesticide use in commercial vegetable gardens and sawah farming (Hoppenreijs and Lith 2016). The consequence of a decrease in bird diversity in rural mountain ecosystem of West Java is to reduce local knowledge of mountain people of West Java. As a result, study on ornithology on birds in the rural mountain of Kertasari area of Upper Citarum Watershed of West Java is considered very important. This location is located at the adjoining area of Mount Gede Pangrango as located in Koningsberger research. Therefore, the bird list of Koningsberger that is based on research at the beginning of the twentieth can be compared to that of our present research based on the ethnoornithology approach. Ethnoornithology, a branch of ethnozoology, is a relatively recent growing academic discipline. This scientific study is on relationships between people and birds in culture, a relationship that often spans prolonged periods (Pam 2017).

The purpose of the study was to account for vernacular or folk names, folk classification, and folkloric birds based on case in the mountain people of Keratasari, Upper Citarum, West Java, Indonesia.

\section{MATERIALS AND METHODS}

\section{Study site}

The field research was conducted in the village of Cikembang, Cihawuk, Neglawangi, and Tarumajaya, of
Kertasari Sub-disrict, Upper Citarum watershed, Bandung District, West Java, Indonesia (Figure 1).

The collected data are part of a larger ethnographic research project on cultural aspects of upland cultivation among the Sundanese in the mountainous region of West Java. The project is being carried out in the eastern section of Pangalengan Plateau, a narrow valley of about 15.000 hectares at over $1400 \mathrm{~m}$ above sea level which is located in the east and southeast of the Upper Citarum watershed and, astronomically, on $107^{0} 37^{\prime} 12^{\prime}$ ' $-107^{0} 44^{\prime} 24$ east longitude dan $07^{\circ} 83^{\prime} 12-07^{0} 39^{\prime} 12$ south latitude. A general landscape of the area consists of small and fragmented portions of flat areas meet with hilly and undulating landscapes dominated by steep and forested mountainous regions, with the cool climate, that ranges from 15-26 centigrade, combines with fertile land make the research site suitable for horticulture and dairy farm activities.

The area is resided by nearly 70.000 inhabitants. Administratively, the Kertasari sub-district can be divided into eight villages, including Cikembang, Cihawuk, Neglawangi, and Tarumajaya. Most of the inhabitants of this valley are peasant and smallholders who grow subtropical vegetables such as kentang/Irish potatoes (Solanum tuberosum L), bawang daun/leeks (Alium pistulosum L), kol/cabbages (Brassica oleracea var capitata), and carrots (Daucus carota var sativus) supplying for local markets (Figure 2). With only $46 \%$ of households who own and have access to land, thus the rest depend on their livelihood as farm laborers. Due to the existence of forests around the valley, the area provides plenty of habitat for birds compared to other regions, and in the end, it creates income opportunities through bird hunting (Hakim et al 2020).
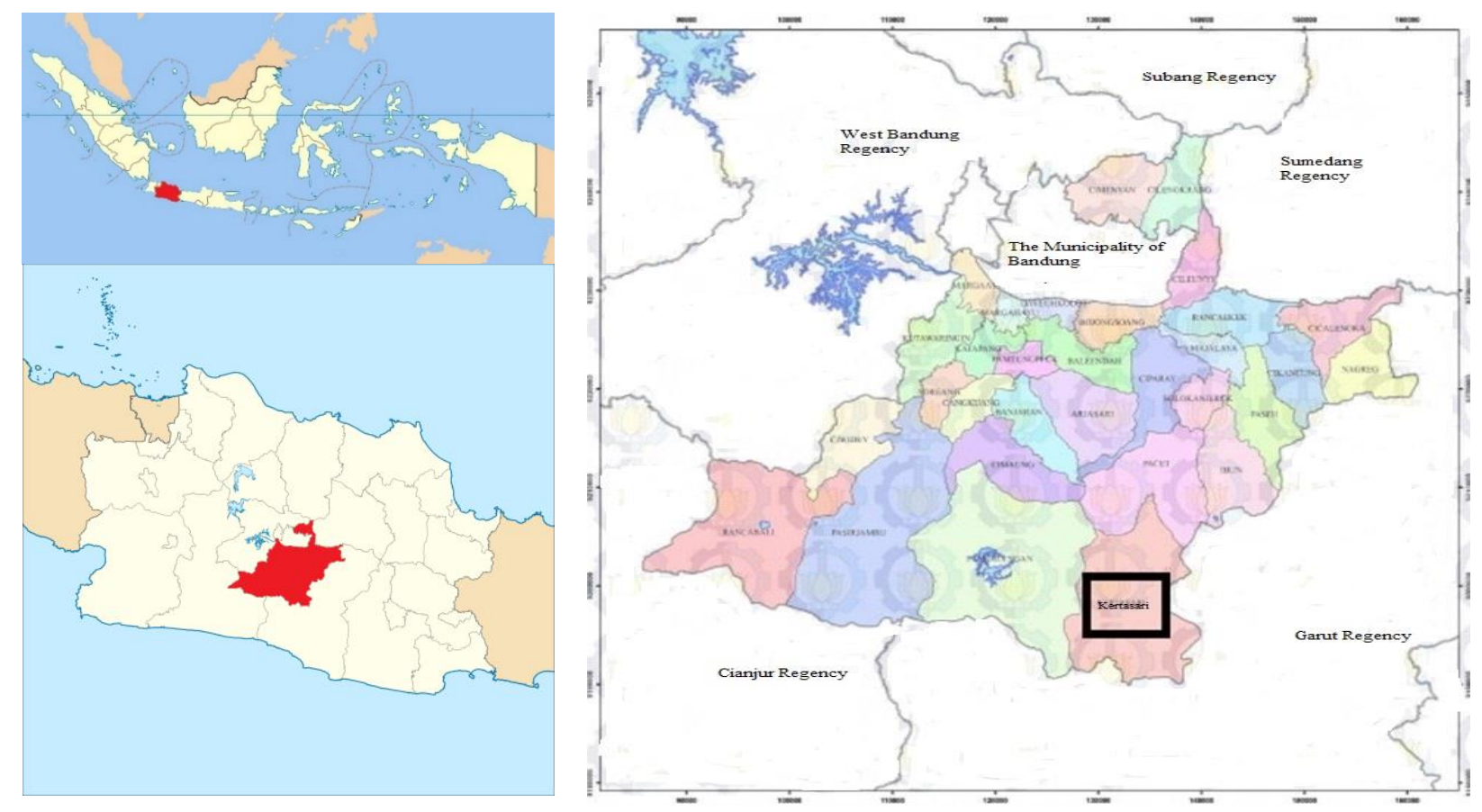

Figure 1. Map of the location of the study area in Kertasari Sub-disrict, Upper Citarum watershed, Bandung District, West Java, Indonesia 


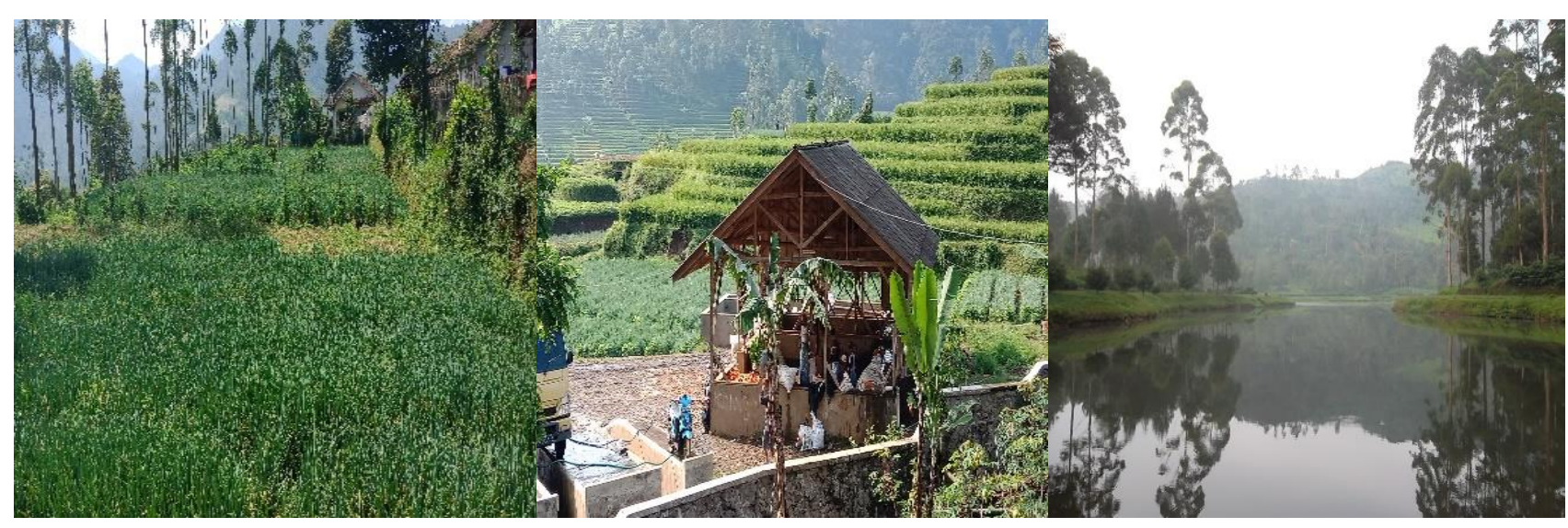

Figure 2. Garden of bawang daun/leek (Allium fistulosum L) (left) and the rice field and scattered trees grow in the hill (middle) can be seen in the background, and relict forest in surrounding of Cisanti lake (right), of Sukasari of Citarum upstream, West Java, Indonesia

\section{Research procedures}

The method used in this study was qualitative with ethnoornithological approach (Iskandar 2007; 2018; Pam 2017). Since the ethical aspects must be considered in the study on ethnoornitological research, consent from the formal leaders and local community must be obtained. I was careful to ensure the right of individual communities were not violating upon. Therefore, the first stage of our research was asking permission from the village leaders as well as sub-district leaders by submitting the formal letter from the university. The second stage, before conducting interview and focus group discussions with informants, was that we introduced our research time, made socialization, and asked permission from informants and local community. Moreover, intensive research activities, including deep interviews and focus group discussions with informants were employed.

Avifauna data, including naming of birds, folk classification, and bird mythologies were collected by deep interviews with informants in four different villages, namely Cikembang, Cihawuk, Neglawangi, and Tarumajaya. The bird naming data were elicited through a systematic importation from colored pictorial representations of birds presented to people. In the same token, should people describe birds by mimicking its voice, we presented them auditoria representation. In all, we communicated in local vernacular, the Sundanese, to share Sundanese/local names of the birds with designated groups. Data were organized to facilitate analysis of particular aspects of folk taxonomic such as contrast, level, and ranking of taxa; hierarchical inclusion and depth in taxonomic structure, grading within categories, taxonomic space, and comparison of folk and scientific taxonomies (Berlin 1992). In this format, data could be compared, contrasted, or combined with scientific (Tidemann et al. 2010; Sinclair et al. 2010) and other ethnobiological data (Alves et al. 2016).

In addition to taxonomic data in the context that we created, folklore data of birds are presented. The folkloric data provide examples of symbolic relationships between bird names and their cultural references (Ellen 1993). Our interlocutors share folklore in the form of stories and reflections about birds' voices and life that are experienced throughout their lives. Moreover, they also contribute folklore in the context of the group elicitation session. Reflecting on two different modalities of data collection and the style in which data are collected, our voices shift as we move between taxonomy and folklore sections.

\section{Data collection procedures and analysis}

We presented 40 colorful plates from MacKinnon's (1988) Field Guide to the Bird of Java and Bali to 12 groups of independent village people. Based on the composition of its members, these groups are divided into two categories: groups of ordinary villagers, which further arranged into eight different groups; and groups of local hunters that clustered into four groups

The design is largely opportunistic for the first category groups, that is, we take advantage of situations that are conducive to data collection and do not try to control group composition based on generation and gender. The aim is to collect data in a way that does not make interference in their daily life. As interesting visual objects, after being introduced and held in their hands, the book becomes part of the conservation. Images of well-designed species and beautifully varied in shape and color make the page layout itself fun and stimulating booster. The origin of book and unintelligence texts (none of them read English) does not hinder them from readability and familiarity, so the book works well as a companion tool. It is a well-received artifact that promotes and coordinates sociality around one or two hours per session

Obtained data from each group represent a consensus of five to eight people. They are a mixture of generation and gender (except for the groups of hunters whose members are all male aged between 20 to 55 years old). Throughout discussions, each group decides on and agrees upon a single name for each bird they identify. Apart from respecting older and knowledgeable people, we found that there are no striking differences in power or authority relations that affect the consensus.

The names of the birds listed here are sequenced in such a way that the relationship between Sundanese and 
scientific names is prominent. Each Sundanese name is listed together with the plate and identification number of each designated species, the appropriate scientific name, a common name in English, collection groups and the total number of groups that make identification and expression of consensus between groups.

Sundanese name that identifies a category is mostly primary lexeme (which cannot be translated directly) and is listed in alphabetical order in table. Binomials that combine a lexeme with a modifier are listed under the main lexeme (for example anis-sisik [scally thrush] is a binomial that will appear under anis [thrush], a lexeme listed alphabetically). In sequencing these categories, different scientific species are identified by the same name and thus are listed from highest to lowest consensus. When consensus ratings are the same, they are listed in the order to which they appeared in the book.

Data which were obtained from interview and focus group discussion with informants were analyzed by crosschecking, summarizing and synthesizing, and making narrative account with descriptive analysis and evaluative (Newing et al. 2011). Cross-checking was done to obtain valid data from different resources, including different informants, reports, and direct observation in the field. The validated data were summarized and synthesized, and then were narrated descriptively, analyzed, and evaluated.

\section{RESULTS AND DISCUSSION}

\section{Vernacular names of birds}

Based on deep interviewed and focus group discussion with informants conducted in Kertasari, upper Citarum Watershed, West Java in 2019 and 2020, 222 bird species were recognized by informants, representing 170 vernacular names (due to some cases, one species of Bird may have several bird vernacular names). Of total 222 species, 93 were recorded by Koningsberger's list (Table 1). In general, the term "Sundanese" refers to people and their language. First, the Sundanese people are known to be the second-largest ethnic group in Indonesia after the Javanese. They are a native inhabitant of the western part of Java island (Minahan 2012). Secondly, it is too the name of a language, a Malayo-Polynesian language, spoken mainly by the people in the western part of Java island. The language can be classified into six main groups of dialects. One of them, the Priangan dialect, is spoken mainly by people who inhabit the mountainous region in the middleto-southern part of western Java (Anderson 1996; MüllerGotama 2001).

As can be seen from Table 1 that bird vernacular names of Sundanese people are based on voice (phononym) (42,94 \%), morphological (morphonym) (20.00\%), behavior or function (ergonym), and feeding guild (phagonym) $(4,70 \%)$ of birds, but some of vernacular names of bird were also unidentified (Table 2).

Based on our study, it can be revealed that some names that are currently popular among villagers are not on the Koningsberger's list (Figure 1). Monomial anis, for example, is identified by all people in all groups but absent in Koningsberger's books. This name specifically refers to Geokichla citrina Latham, Family of Turdidae which is named manuk-cacing [manuk=bird, cacing=ground worm] by Koningsberger's informant. The term manuk-cacing also known by six groups as an alternative for anis. Possibly, anis are imported from Indonesia's official name that popular among bird traders and keepers (Iskandar 2017). No single person in data collection groups knows its meaning. Anis and around 60 other binomials belong to names whose meanings are unknown. Some of them, such as dadali [Falconidae], heulang [Accipitridae], bango [Egret], trik [Bee-eater], manyar [Ploceus manyar Horsfield, Family of Ploceidae], and pelung [Porphyrio porphyrio Linnaeus, Fairy of Rallidae] are an ancient name for the same bird family, genus, or species that can be found in a $9^{\text {th }}$ century Old Javanese manuscripts, Kakawin Ramayana (see Mulyanto et al. 2019).

Among the identified monomials, most Sundanese names of birds can be categorized as phononym or onomatopoeic. Name for Gallinago stenura Bonaparte, Family of Scolopacidae, for example, is belekék which resembles a bird's distinctive voice. Similarly, the term tuweuw for Eudynamys scolopaceus Linnaeus, Family of Cuculidae, culik-culik for Psilopogon australis Horsfield, Family of Megalaimidae, ékék for Psittacula alexandri Linnaeus, Family of Psittacidae, and jogjog for Pycnonotus goiavier Scopoli, Family of Pycnonotidae.

Some monomials are morphonym, especially based on plumage, colors, and physical characteristics of bird's form. For example, the name for Pericrocotus flammeus Foster, Family of Campephagidae is sepah that is inclined more toward bird's distinctive orange color that resembles the color of areca nut (also seupah in Sundanese), And also sapu for Fantail [Rhipiduridae].

Some monomials are phagonymic. For example, sèsèpmadu, which refers to Sunbird [Nectariniidae], literally means 'honey or nectar sipper'.

Modifiers in binomials are mostly bionymic and morphonymic. For example, modifiers in binomial kicuitleuweung [Dendronanthus indicus Gmelin, Family of Motacillidae] and kicuit-sawah [Anthus rufulus Vieillot, Family of Motacillidae] are bionymic ('leuweung' means forest and 'sawah' means paddy-field), meanwhile modifiers in saéran-gunting [Dicrurus macrocercus Vieillot, Family of Dicruridae] and saéran-hawu [Dicrurus leucophaeus Vieillot, Family of Dicruridae] are morphonymic ('gunting' means scissor, related to the tail shape, and 'hawuk' means ash, related to the color).

Table 2. The meaning of the bird's vernacular name in rural Sundanese people of Kertasari of West Java, Indonesia

\begin{tabular}{lcc}
\hline $\begin{array}{c}\text { Meaning of vernacular } \\
\text { names of birds }\end{array}$ & $\begin{array}{c}\text { Species } \\
\text { number }\end{array}$ & $\begin{array}{c}\text { Percent of the } \\
\text { total }\end{array}$ \\
\hline Phononym & 73 & 42.94 \\
Morphonym & 34 & 20.00 \\
Ergonym & 16 & 9.41 \\
Bionym & 14 & 8.23 \\
Phagonym & 8 & 4.70 \\
Unidentified & 25 & 14.70 \\
Total & 170 & 100.00 \\
\hline
\end{tabular}


Table 1. Various vernacular names of birds were documented based on rural Sundanese people of Kertasari of West Java, Indonesia

\begin{tabular}{|c|c|c|c|c|}
\hline Sundanese name & Scientific name \& Family [English common name] & 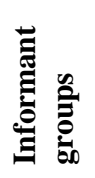 & 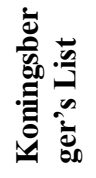 & Meaning \\
\hline Anis & Geokichla citrina Latham, Turdidae [Orange-headed Trush] & 12 & - & Unidentified \\
\hline Anis-awi & Turdus obscurus Gmelin, Turdidae [Eyebrowed Thrush] & 7 & - & Bionym \\
\hline Anis-kembang & Geokichla interpres Temminck, Turdidae [Chestnut-capped Thrush] & 9 & - & Morphonym \\
\hline Anis-héjo & Cyornis unicolor Blyth, Muscicapidae [Pale blue flycatcher] & 6 & - & Morphonym \\
\hline Anis-sibéria & Geokichla sibirica Pallas, Turdidae [Siberian Thrush] & 7 & - & Bionym \\
\hline \multirow{2}{*}{ Anis-sisik } & Zoothera dauma Latham, Turdidae [Scaly Thrush] & 9 & - & Morphonym \\
\hline & Zoothera andromeda Temminck, Turdidae [Sunda Thrush] & 4 & & \\
\hline Apung & Mirafra javanica Horsfield, Alaudidae [Horsfield's Bushlark] & 4 & + & Ergonym \\
\hline \multirow[t]{2}{*}{ Bango } & Egretta alba Linnaeus, Ardeidae [Great Egret] & 12 & + & Unidentified \\
\hline & Egretta garzetta Linnaeus, Ardeidae [Little Egret] & 12 & & \\
\hline Bango-blewok & Mycteria cinerea Raffles, Ciconiidae [Milky Stork] & 9 & - & Morphonym \\
\hline Bango-hideung & Ciconia episcopus Boddaert, Ciconiidae [Woolly-necked Stork] & 4 & - & Morphonym \\
\hline Bango-tongtong & Leptoptilos javanicus Horsfield, Ciconiidae [Lesser Adjutant] & 4 & - & Morphonym \\
\hline Bébéak & Eurystomus orientalis Linnaeus, Coraciidae [Oriental Dollarbird) & 4 & + & Phononym \\
\hline Belekék & Gallinago stenura Bonaparte, Scolopacidae [Pin-tailed Snipe) & 4 & + & Phononym \\
\hline Belekék-bulan & Hydrophasianus chirurgus Scopoli, Jacanidae [Pheasant-tailed Jacana] & 4 & - & Morphonym \\
\hline Belekék-kembang & $\begin{array}{l}\text { Rostratula benghalensis Linnaeus, Rostratulidae [Greter Painted } \\
\text { Snipe] }\end{array}$ & 4 & + & Morphonym \\
\hline Belekok & Ardeola speciosa Horsfield, Ardeidae [Javan Pond Heron] & 12 & + & Phononym \\
\hline \multirow{2}{*}{ Bencé } & Turnix suscitator Gmelin, Turnicidae [Barred Buttonquail] & 12 & + & Phononym \\
\hline & Turnix sylvaticus Desfontaines, Turnicidae [Small Buttonquail] & 10 & & \\
\hline \multirow[t]{2}{*}{ Bencér } & Cacomantis merulinus Scopoli, Cuculide [Plaintive Cuckoo] & 5 & - & Phononym \\
\hline & Cacomantis sonneratii Latham, Cuculidae [Banded Bay Cuckoo] & 2 & & \\
\hline Berekesését & Vanellus macropterus Wagler, Charadriidae [Javan Lapwing] & 2 & & Ergonym \\
\hline Bincarung & Oriolus chinensis Linnaeus, Oriolidae [Black-naped Oriole] & 4 & + & Unidentified \\
\hline Beureum-gado & $\begin{array}{l}\text { Dicaeum sanguinolentum Temminck, Dicaeidae [Blood breasted } \\
\text { Flowerpecker] }\end{array}$ & 6 & + & Morphonym \\
\hline Boroboy & Eurylaimus javanicus Horsfield, Eurylaimidae [Branded Broadbill] & 2 & - & Phononym \\
\hline Brek-brek & $\begin{array}{l}\text { Napothera epilepidota Temminck Pellorneidae (Eyebrowed Wren } \\
\text { Babbler] }\end{array}$ & 3 & + & Phononym \\
\hline \multirow[t]{3}{*}{ Bueuk } & Otus lempiji Horsfield, Strigidae [Sunda Scops Owl] & 12 & + & Phononym \\
\hline & Otus rufescens Horsfield, Strigidae [Reddish Scop-Owl] & 11 & & \\
\hline & Otus angelinae Finsch, Strigidae [Javan Scops Owl] & 6 & & \\
\hline Bultok & Psilopogon lineatus Vieillot, Megalaimidae [Lineated Barbet] & 4 & + & Phononym \\
\hline Bututut & $\begin{array}{l}\text { Psilopogon corvinus Temminck, Megalaimidae [Brown-throated } \\
\text { Barbet] }\end{array}$ & 7 & - & Phononym \\
\hline \multirow[t]{2}{*}{ Caladi } & Chrysocolaptes guttacristatus Tickell, Picidae [Greater Flameback] & 12 & + & Unidentified \\
\hline & Dinopium javanense Ljungh, Picidae [Common Flameback] & 11 & & \\
\hline Caladi-batu & Meiglyptes tristis Horsfield, Picidae [Buff-Rumped Woodpecker] & 5 & - & Morphonym \\
\hline Caladi-kundang & Dinopium javanense Horsfield, Picidae [Common Flameback] & 8 & + & Morphonym \\
\hline \multirow[t]{2}{*}{ Caladi-lumut } & Sitta frontalis Swainson, Sittidae [Velvet Fronted Nuthatch] & 4 & - & Morphonym \\
\hline & Sitta azurea Lesson, Sittidae [Blue Nuthatch] & 2 & & \\
\hline Caladi-muncang & Picoides moluccensis Gmelin, Picidae [Sunda Pygmy Woodpecker] & 4 & - & Morphonym \\
\hline Camperling & Aplonis panayensis Scopoli, Strunidae [Assian Glossy Starling] & 4 & + & Morphonym \\
\hline Cangéhgar & Gallus varius Shaw, Phasianidae [Green Junglefowl] & 12 & - & Phononym \\
\hline Cangkurawok & $\begin{array}{l}\text { Psilopogon armillaris Temminck, Megalimidae [Flame-Fronted } \\
\text { Barbet] }\end{array}$ & 4 & + & Phononym \\
\hline \multirow[t]{2}{*}{ Cangkurileung } & Pycnonotus aurigaster Vieillot, Pycnonotidae [Sooty headed Bulbul] & 12 & + & Phononym \\
\hline & $\begin{array}{l}\text { Pycnonotus bimaculatus Horsfield, Pycnonotidae [Orange-spotted } \\
\text { Bulbul] }\end{array}$ & 10 & & \\
\hline Carawak & Pycnonotus zeylanicus Gmelin Pycnonotidae [Tiger Shrike] & 4 & - & Phononym \\
\hline Cendét & Lanius tigrinus Drapiez, Laniidae [Tiger Shrike] & 4 & - & Phononym \\
\hline Cerorot & $\begin{array}{l}\text { Hemipus hirundinaceus Temminck, Campephadae [Black-winged } \\
\text { Flycatcher-shrike] }\end{array}$ & 4 & - & Phononym \\
\hline Cèt-gunggung & Cuculus lepidus Müller, Cuculidae [Sunda Cockoo] & 12 & - & Phononym \\
\hline Ciang-ciang & Orthotomus sutorius Pennant, Cisticolidae [Common Tailorbird] & 5 & - & Phononym \\
\hline \multirow[t]{2}{*}{ Cicangkoréng } & Megalurus palustris Horsfield, Locustellidae [Striated Grassbird] & 4 & - & Phononym \\
\hline & Locustella certhiola Pallas, Locustellidae [Lanceolated Warbler] & 3 & & \\
\hline
\end{tabular}


Cici

Cicing-goléng

Cinétnét

Cingcoang

Cipeuw

Ciung

Cukahkéh

Cuhcur

Culik-culik

Dadali

Dadali-leutik

Dècu/Dèdècu

Dederuk

Dudut

Dudut-troktok

Ékék

Dudut-candung

Erow

ékék-gèléng

Gagak

Galatik

Gaok

Gasngék

Goléjra

Hahayaman

Haur

Heulang

$\quad$ Heulang-kutuk
Hingkik
Jijiprék
Japati
Jingjing-teureup

Jogjog

Julang

Kacamata

Kacer

Kalacés

Kangkaréng

Kapinis
Locustella lanceolata Temminck, Locustellidae [Lanceolated Warbler] Prinia polychroa Temminck, Cisticolidae [Brown Prinia]

Cyornis banyumas Horsfield, Muscicapidae [Javan Blue-Flycatcher]

Orthotomus ruficeps Lesson, Cisticolidae [Ashy Tailorbird]

Orthotomus sepium Horsfield, Cisticolidae [Olive-backed Tailorbird]

Brachypteryx leucophris Temminck, Muscicapidae [Lesser Shortwing]

Brachypteryx montana Horsfield, Muscicapidae [White-browed

Shortwing]

Aegithina tiphia Linnaeus Aegithinidae [Common Iora]

Myophonus caeruleus Scopoli, Muscicapidae [Blue Whistiling-Thrush]

Myophonus glaucinus Temminck, Muscicapidae [Javan Whistling-

Thrush]

Todiramphus chloris Boddaert, Alcedinidae [Collared Kingfisher]

Lacedo pulchella Horsfield, Alcedinidae [Banded Kingfisher]

Alcedo atthis Linnaeus, Alcedinidae [Common Kingfisher]

Caprimulgus pulchellus Salvadori, Caprimulgidae [Salvadori's

Nightjar]

Caprimulgus indicus Latham, Caprimulgidae

Caprimulgus macrurus Horsfield, Caprimulgidae [Large-tailed

Nightjar]

Caprimulgus affinis Horsfield, Caprimulgidae [Savanna Nightjar]

Psilopogon australis Horsfield, Megalainidae [Little Barbet]

Falco peregrinus Tunstall, Falconidae [Peregrine Falcon]

Falco moluccensis Bonaparte, Falconidae [Spotted Kestrel]

Falco severus Horsfield, Falconidae [Oriental Hobby]

Falco subbuteo Linnaeus, Falconidae [Eurasian Hobby]

Microhierax fringillarius Drapiez, Falconidae [Black Thigh Falconet]

Saxicola caprata Linnaeus, Muscicapidae [Pied Bush Chat]

Streptopelia bitorquata Temminck, Columbidae [Island Collared

Dove]

Zanclostomus javanicus Horsfield, Cuculidae [Red-billed Malkoha]

Centropus sinensis Stephen, Cuculidae [Greater Coucal]

Centropus nigrorufus Cuvier, Cuculidae [Sunda Coucal]

Psittacula alexandri Linnaeus, Psittacidae [Red-breasted Parakeet]

Cissa thalassina Temminck, Corvidae [Javan Green-Magpie]

Ficedula mugimaki Temminck, Muscicapidae [Mugimaki Flaycatcher]

Ficedula zanthopygia Hay, Muscicapidae [Yellow-rumped

Flaycatcher]

Corvus enca Horsfield, Corvidae [Slender billed-Crow]

Lonchura oryzivora Linnaeus, Estrildidae [Java sparrow]

Corvus macrorhynchos Wagler, Corvidae [Large-billed Crow]

Lacedo pulchella Horsfield, Alcedinidae [Banded Kingfisher]

Passer montanus Linnaeus, Passeridae [Eurasian Tree Sparrow]

Gallicrex cinerea Gmelin, Rallidae [Watercock]

Lewinia striata Linnaeus, Rallidae [Slaty-breasted Rail]

Amaurornis phoenicurus Pennant, Rallidae [White breasted water hen]

Copsychus saularis Linnaeus, Muscicapidae [Oriental-magpie Robin]

all species of Accipitridae, extended to Pandionidae

Haliastur indus Boddaert, Accipitridae [Brahminy Kite]

Strix leptogrammica Temminck, Strigidae [Brown Wood Owl]

Ketupa ketupu Horsfield, Strigidae [Buffy Fish-Owl]

Prinia flaviventris Delessert, Cisticolidae [Yellow-bellied Prinia]

Columba livia Gmelin, Columbidae [Rock Dove]

Hemipus hirundinaceus Temminck, Campephagidae [Black-winged

Flaycatcher-shrike]

Pycnonotus goiavier Scopoli, Pycnonotidae [Yellow-veted Bulbul]

Rhyticeros undulatus Shaw, Bucerotidae [Wreated Hornbill]

Zosterops flavus Horsfield, Zosteropidae [Javan White-eye]

Zosterops montanus Bonaparte, Zosteropidae [Mountain White-eye]

Zosterops palpebrous Temminck, Zosteropidae [Indian White-eye]

Zosterops chloris Bonaparte, Zosteropidae [Lemon-bellied White-eye]

Copsychus saularis Linnaeus, Turdidae [Oriental Magpie-robbin]

Arachnothera affinis Horsfield, Nectariniidae [Streaky-breasted

Spiderhunter]

Anthracoceros albirostris Shaw, Bucerotidae [Oriental Pied Hornbill]

Apus nipalensis Hodgson, Apodidae [House swift]

Collocalia esculenta Linnaeus, Apodidae [Glossy Swoftlet]
3

6

4

12

9

6

4

12

12

10

12

- Phononym

$+\quad$ Phononym

- Phononym

$+\quad$ Phononym

+ Phononym $+\quad$ Phononym

- Unidentified

- Morphonym

- Unidentified

+ Phononym

+ Phononym

+ Phononym

$+\quad$ Morphonym

+ Phononym

+ Phononym

- Unidentified

+ Phononym

+ Phonomym

+ Phononym

+ Phononym

- Unidentified

- Morphonym

- Unidentified

+ Ergonym

- Unidentified

+ Ergonym

- Phononym

- Unidentified

- Bionym

+ Phononym

+ Unidentified

- Morphonym

- Phononym

+ Phononym

$+\quad$ Unidentified

$+\quad$ Unidentified 


\author{
Kapinis-guha \\ Kapinis-bélang \\ Kasintu \\ Kékés \\ Keling \\ Kérak \\ Kicuit \\ Kicuit-leuweung \\ Kicuit-kebo \\ Kicuit-sawah \\ Kokokan \\ Kokok-beluk \\ Koréak \\ Korés \\ Kuak \\ Kucica \\ Kundul \\ Kuwik-kuwik \\ Langgir \\ Lontrok \\ Luntur \\ Mancirang \\ Maninting \\ Mangadeuh \\ Manuk-beusi
}

Manuk-buah

Manuk-cacing

Manuk-daun

Manuk-hurang

Manuk-uncal

Manuk-kaso

Manuk-kopi

Manyar

Manyar-emas

Merak

Ongklet

Pacikrak

Panganten

Paok

Pecampur

Peking

Pelung

Pergum

Petulak

Prukutut

Piit

Pijantung

Poksai

Puter

Puyuh-gonggong
Hirundo rustica Linnaeus, Hirundinidae [Barn Swallow]

Aerodramus fuciphagus Thunberg, Apodidae [Edible-nest Swiftlet]

Cecropis striolata Schlegel, Hirundinidae [Striated Swallow]

Gallus gallus Linnaeus, Phasianidae [Red Junglefowl]

Anthreptes malacensis Scopoli Nectariniidae [Brown-throated Sunbird]

Aplonis panayensis Scopoli, Strunidae [Asian Glosy Starling]

Acridotheres javanicus Cabanis, Sturnidae [Javan Myna]

Acridotheres melanopterus Daudin, Sturnidae [Black-winged Starling]

Cinnyris jugularis Linnaeus, Nectariniidae [Olive-backed Sunbird]

Dendronanthus indicus, Gmelin Motacillidae [Forest Wagtail]

Motacilla flava Linnaeus, Motacillidae [Western Yellow Wagtail]

Anthus rufulus Vieillot, Motacillidae [Paddyfield Pipit]

Ixobrychus eurhythmus Swinhoe, Ardeidae [Schrenk's Bittern]

Strix leptogrammica Temminck, Strigidae [Brown Wood Owl]

Tyto alba Scopoli, Strigidae [Barn Owl]

Alophoixus bres Lesson, Pycnonotidae [Grey-cheeked Bulbul]

Nycticorax nycticorax Linnaeus, Ardeidae [Black-crowned Night-

Heron]

Kittacincla malabarica Scopoli, Muscicapidae [White Rumped Shama]

Bubulcus ibis Linnaeus, Ardeidae [Cattle Egret]

Surniculus lugubris Horsfield, Cuculidae [Square tailed drongo

cuckoo]

Merops leschenaulti Vieillot, Meropidae [Chestnut-headed Bee-eater]

Zanclostomus javanicus Horsfield, Cuculidae [Red-billed Malkoha]

Harpactes oreskios Temminck, Trogonidae [Orange-bresated Trogon]

Pycnonotus plumosus Blyth, Pycnonotidae [Olive winged-Bulbul]

Alcedo meninting Horsfield, Alcedinidae [Blue-eared Kingfisher]

Anthreptes malacensis Scopoli, Nectariniidae [Plain-throated Sunbird]

Halcyon cyanoventris Vieillot, Alcedinidae [Javan Kingfisher]

Halcyon coromanda Latham, Alcedinidae [Ruddy Kingfisher]

Halcyon smyrnensis Linnaeus, Alcedinidae [White-throated

Kingfisher]

Artamus leucorynchus Linnaeus, Artamidae [White-breasted

Woodswallow]

Geokichla citrina Latham, Turdidae [Orange-headed Thrush]

Chloropsis cochinchinensis Gmelin, Chloropseidae [Blue-winged

Leafbird]

Alcedo atthis Linnaeus, Alcedinidae [Common Kingfisher]

Alcedo coerulescens Vieillot, Alcedinidae [Small Blue Kingfisher]

Alcedo euryzona Temminck, Alcedinidae [Blue-banded Kingfisher]

Macropygia unchall Wagler, Columbidae [Barred Cuckoo-dove]

Macropygia emiliana Bonaparte, Columbidae [Ruddy Cuckoo-dove]

Macropygia ruficeps Temminck, Columbidae [Little Cuckoo-Dove]

Timalia pileata Horsfield, Timaliidae [Chestnut-capped Babbler]

Pomatorhinus montanus Horsfield, Timaliidae [Chestnut-backed

Scimitar-Babbler]

Ploceus manyar Horsfield, Ploceidae [Streaked Weaver]

Ploceus hypoxanthus Sparrman, Ploceidae [Asian golden weaver]

Ploceus philippinus Linnaeus, Ploceidae [Baya Weaver]

Pavo muticus Linnaeus, Phasianidae [Green peafowl]

Platylophus galericulatus Cuvier, Platylophidae [Crested jay]

Prinia familiaris Horsfield, Cisticolidae [Bar-winged Prinia]

Metopidius indicus Latham, Jacanidae [Bronze-winged Jacana]

Hydrornis guajanus Müller, Pittidae [Javan Banded-Pitta]

Pycnonotus dispar Horsfield, Pycnonotidae [Ruby-throated Bulbul]

Lonchura punctulata Linnaeus, Estrildidae [Sacaly-bresated Munia]

Porphyrio porphyrio Linnaeus, Rallidae [Purple Swampen]

Ducula aenea Linnaeus, Columbidae [Green Imperial-pigeon]

Tephrodornis virgatus Temminck, Campephagidae [Large

Woodshrike]

Geopelia striata Linnaeus, Columbidae [Zebra Dove]

Lonchura leucogastroides Moore, Estrildidae [White- bellied Munia]

Arachnothera longirostra Latham, Nectariniidae [Liitle Spiderhunter]

Garrulax rufifrons Lesson, Leiotrichidae [Rufous-fronted

Laughingthrush]

Streptopelia bitorquata Temminck, Columbidae [Sunda collared-dove] 12

Arborophila orientalis Horsfield, Phasianidae [Grey-breasted Partidge] 12
- Bionym

- Morphonym

$+\quad$ Phononym

Phononym

+ Morphonym

- Phononym

$+\quad$ Phononym

- Bionym

+ Bionym

Bionym

Phononym

+ Phononym

Phononym

Phnonym

Phononym

$+$

Unidentified

Unidentified

Phononym

- Ergonym

+ Morphonym

- Morphonym

$+\quad$ Unidentified

- Unidentified

$+\quad$ Ergonym

$+\quad$ Morphonym

$+\quad$ Unidentified

$+\quad$ Phagonym

+ Morphonym

$+\quad$ Phagonym

+ Ergonym

+ Bionym

+ Bionym

$+\quad$ Unidentified

- Morphonym

Bionym

$+\quad$ Unidentified

+ Phagonym

+ Phononym

Unidentified

$+\quad$ Phogonym

+ Unidentified

+ Phogonym

+ Morphonym

+ Unidentified

$+\quad$ Monophonym

- Phononym

+ Phononym

Phagonym

- Unidentified

$+\quad$ Phononym

+ Phononym 
Raja-udang

Rangkong

Saéran

\section{Saéran-batu}

Saéran-bodok

Saéran-gelo

Saéran-gunting

Saéran-hawu

Sapu

$\quad \begin{aligned} & \text { Sapu-beureum } \\ & \text { Seupah }\end{aligned}$
Sering
Sesep-madu

Sier

Sngséwan

Siuh

Téngtélok

Tepus

Téték-réyod

Tikukur

Tilil

Tinggal-anak

Titihan

Titimplik
Titiran
Tohtor
Trik
Towéd
$\quad$ Towéd-leuncang
Tulang-kuwiwi
Tulung-tumpuk
Tuweuw
Uncuing/siit-uncuing
Ungkut-ungkut
Walét
Walét-curug
Walik

Walik-kembang

Waliwis

Werweg
Arborophila javanica Gmelin Phasianidae [Chestnut-bellied Partridge] Alcedo coerulescens Vieillot, Alcedinidae [Small Blue Kingfisher] Alcedo euryzona Temminck, Alcedinidae [Blue-banded Kingfisher] Buceros rhinoceros Linnaeus, Bucerotidae [Rhinoceros Hornbill] Dicrurus annectans Hodgson, Dicruridae [Crow-billed Drongo] Dicrurus hottentottus Linnaeus, Dicruridae [Hair-crested Drongo] Dicrurus remifer Temminck, Dicruridae [Lesser Racket-tailed drongo] Dicrurus paradiseus Linnaeus, Dicruridae [Greater Racket-tailed Drongo]

Crypsirina temia Daudin, Corvidae [Racket-tailed Treepie]

Dicrurus macrocercus Vieillot, Dicruridae [Black Drongo]

Dicrurus leucophaeus Vieillot, Dicruridae [Ashy Drongo]

Rhipidura javanica Sparrman, Rhipiduridae [Malaysian Pied Fantail] Rhipidura perlata Müller, Rhipiduridae [Spotted Fantail]

Rhipidura euryura Müller, Rhipiduridae [White-bellied Fantail]

Rhipidura phoenicura Müller, Rhipiduridae [Rufous-tailed Fantail]

Pericrocotus flammeus Foster, Campephagidae [Flame Minivet] Pericrocotus miniatus Temminck, Campephagidae [Sunda Minivet] Chrysocorythus estherae Finsch, Fringillidae [Mountain Serin] Aethopyga siparaja Raffles, Nectariniidae [Crimson Sunbird] Aethopyga eximia Horsfield, Nectariniidae [White-Flanked Sunbird] Aethopyga mystacalis Temminck, Nectariniidae [Javan Sunbird] Apalharpactes reinwardtii Temminck, Trogonidae [Javan Trogon] Saxicola caprata Linnaeus, Muscicapidae [Pied Bush Chat] Cyornis banyumas Horsfield, Muscicapidae [Hill Blue-flycatcher] Pycnonotus plumosus Blyth, Pycnonotidae [Olive-winged Bulbul] Stachyris thoracica Temminck, Timaliidae [White-bibbed Babbler] Turdus poliocephalus Latham, Turdidae [Island Thrush] Streptopelia chinensis Scopoli, Columbidae [Spotted Dove] Calidris ferruginea Pontoppidan, Scolopacidae [Curlew Sandpiper]

Calidris ruficollis Pallas, Scolopacidae [Red-Necked Stint] Cuculus canorus Linnaeus, Cuculidae [Common Cuckoo]

Tachybaptus novaehollandiae Stephens, Podicipedidae [Australasian Grebe]

Tachybaptus ruficollis Pallas, Podicipedidae [Little Grebe]

Mirafra javanica Horsfield, Alaudidae [Horsfield's Bushlark]

Geopelia striata Linnaeus, Columbidae [Zebra Dove]

Psilopogon armillaris Temminck, Megalaimidae [Flame-fronted Barbet]

Merops philippinus Linnaeus, Meropidae [Blue-tailed Bee-eater]

Merops leschenaulti Vieillot, Meropidae [Chestnut-headed Bee-eater]

Lanius schach Linnaeus, Laniidae [Long-tailed Shrike]

Lanius cristatus Linnaeus, Laniidae [Brown Shrike]

Phodilus badius Horsfield, Tytonidae [Oriental Bay-Owl]

Psilopogon javensis Horsfield, Megalaimidae [Black-banded Barbet]

Eudynamys scolopaceus Linnaeus, Cuculidae [Asian Koel]

Cacomantis sepulcralis Vigor \& Horsfield, Cuculidae [Rusty Breasted Cuckoo]

Psilopogon haemacephalus Müller, Megalaimidae [Coppersmith

Barbet]

Aerodramus vulcanorum Stresemann, Apodidae [Volcano Swiftlet]

Hydrochous gigas Hartert \& Butler, Apodidae [Giant Swiftlet]

Ramphiculus jambu Gmelin, Columbidae [Jambu Fruit-Dove]

Ptilinopus porphyreus Temminck, Columbidae [Pink-headed Fruit-

Dove]

Ptilinopus melanospilus Salvadori, Columbidae [Black-naped Fruit-

Dove]

Dendrocygna javanica Horsfield, Anatidae [Lesser Whistling Duck]

Dendrocygna arcuata Horsfield, Anatidae [Wandering Whistling

Duck]

Heleia javanica Horsfield, Zosteropidae [Javan Grey-throated Whiteeye]
12

4

$+\quad$ Unidentified

+ Bionym

+ Bionym

$+\quad$ Ergonym

- Morphonym

- Morphonym

+ Morphonym

- Morphonym

+ Morphonym

- Unidentified

+ Phagonym

Unidentified

Ergonym

Ergonym

Phononym

Ergonym

Phononym

Phononym

Phononym

Ergonym

+ Unidentified

- Ergonym

- Ergonym

- Ergonym

$+\quad$ Unidentified

+ Phononym

- Bionym

+ Ergonym

$+\quad$ Phononym

- Phononym

- Phononym

$+\quad$ Phononym

- Uniedentified

- Bionym

+ Morphonym

6

$3+$ Morphonym

12 - Unidentified

Note: Phononym: based on voice; Morphonym: based on morphology; Ergonym: based on function/behavior; Bionym: based on binomial; Phagonym: based on dietary (feeding guild); +: present in Koningsberger's list, -: absent in Koningsberger's list. 


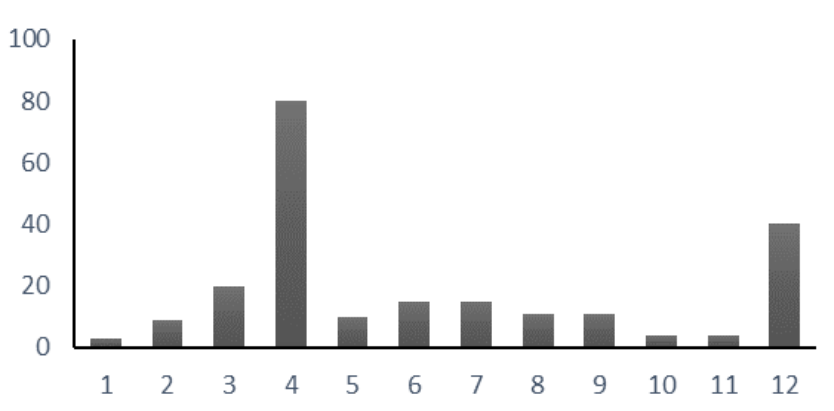

Figure 3. The number of bird names identified by12 group of village people of Kertasari, Upper Citarum Watershed, West Java, Indonesia

Like Sundanese, the Javanese people of Yogyakarta also have various popular folk names of birds based on distinctive voice, color, and morphological. For example, Eudynamis scolopaceus, Sturnus melanopterus, and Dicrurus macrocercus are culturally known as culik-culik, jalak puteh, and srigunting, respectively, which is based on distinctive voice, color, and morphology of birds (Iskandar et al. 2020).

Similarly, some vernacular names of birds in the Philippines, including 'pitupi' (Cacomantus merulinus), 'tuao' (Eudynamys scolopacea), 'tonguitok' (Psilopogon haemacephalus), 'wik wik' (Coracina striata), 'sina-cacao' (Dicrurus balicassius), 'pato-dilao' (Oriolus chinensis), 'bales gugu' (Irena cyanogastra), 'wak-wak' (Corvus enca), and 'rek-rek' (Lanius cristatus) are named among Agta community of Northern Luzon, based on distinctive voice or sound of bird (Van Der Ploeg and Van Weerd 2010).

On the basis of 170 vernacular names of birds, seabirds were barely recorded. There is only one name, titihan, which is also on Koningsberger's list, which refers to grebes [Podicipedidae]. This is because the land use in the study area is predominantly by upland agriculture system, plantation and forest instead of sawah or sea ecosystem.

Based on the consensual degree, only 40 names were identified by all designated groups of villagers, 80 names were identified by only four groups of hunters (Figure 3 ).

Further analysis of these figures needs to be done, however, we skip it for a while since we still need additional information. For instance, concerning local knowledge of village people on birds can be fascinatingly studied for the near future based on age, gender, main livelihood or subsistence practice, bilingualism, and education of the respondent. This is because some studies have recalled that the distribution of knowledge within human communities is not homogenous. For example, based on study on local knowledge on birds of Mushere people of Nigeria, it can be revealed that the local knowledge of respondents statistically differed by village, occupation, and gender (Pam 2017). This is because the predominant mode of local knowledge transmission in rural communities is usually through oral means, including practice and observation. In terms of observation, for example, most rural communities of Kertasari, Upper Citarum, West Java, have differently seen and heard voices of birds among age, gender, and occupation. As can be seen from Figure 3, group of bird hunter have been able to identify 80 bird names because they have frequently observed birds when they hunting birds in the forest.

In addition, concerning the vernacular bird names, it can have revealed that the more someone observed birds in local environment, the more popular will be recognized by the rural community. In other words, many birds have been popularly known by village people because these birds have predominant in their village. Conversely, the rare bird species of their village have been barely known by the village people (Iskandar et al. 2016). Consequently, since many bird populations have dramatically decreased in villages due to many factors, including habitat loss and illegal bird hunting, the local knowledge of village is lower.

Based on our field research, it was recorded some groups of birds that are most often hunted for sale, especially bird of prey (Accipitridae, Falconidae, Strigidae); chirping birds (especially Thrush), birds with beautiful plumage and color, and rare avifauna such as Gallus varius Shaw and Pavo muticus. In addition, there are two groups of birds that are most often hunted for consumption namely wild species of Columbidae and Arborophila. However, there is no habit among villagers to consume bird eggs. Among the various species of birds that are commonly caught by hunters in Kertasari, West Java, are bueuk (Ottus lempiji), anis hejo (Cyornis unicolor), dadali (Falco moluccensis), puyuh gong-gong (Arborophila javanica), koréak (Tyto alba), siér/luntur gunung (Harpactes reinwardtii), tikukur (Streptopelia chinensis); and delimukan (Chalcophaps indica), and these are presented in Figure 4.

\section{Classification of Sundanese and scientific bird names}

Some interesting comparisons can be made between folk and scientific taxonomy. While in many cases, one Sundanese name exactly or almost exactly refers to a scientific genus or species (e.g. 'kacamata' with Zosterops, 'cangéhgar' with Gallus varius Shaw, Family of Phasianidae), in many other cases there are different types of fitness. So, for example, there is only one name for all Falconidae ('dadali') whereas the scientific taxonomy on the bird on Java island breaks this bird family down into two genera and six species (MacKinnon 1988).

Besides, not all binomials under the same lexeme refer to the same genus or even family of birds. For example, under the category of anis (Thrush), there are five binomials, in which four of these refer to other birds in Thrush family, but one binomial, anis-héjo [literally means 'green-anis'], refers to a Flycatcher. Under the category of bango [Ardeidae], there are three other binomials that refer to three species of the Ciconiidae family. The same thing happened for one binomial under the category of caladi. While the term caladi and three binomials under this category refer to members of Picidae family, the binomial caladi-lumut instead refers to two species of Nuthatch [Sittidae]. The physical attributes necessary for systematizing these birds from an evolutionary point of view are not relevant to Sundanese speakers. 


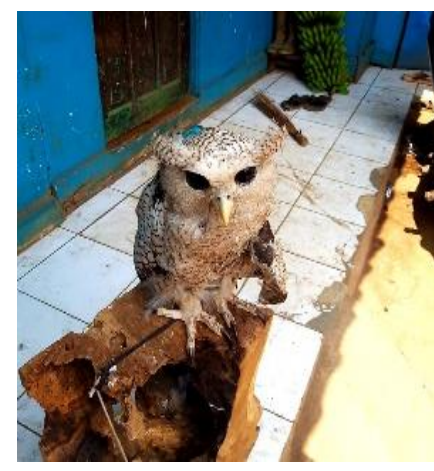

A

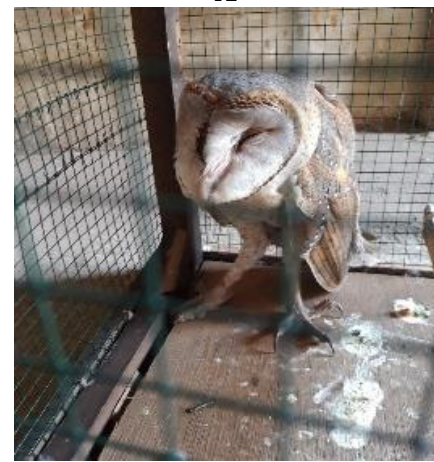

$\mathbf{E}$

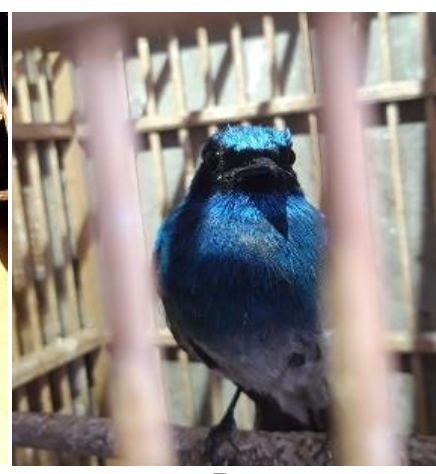

B

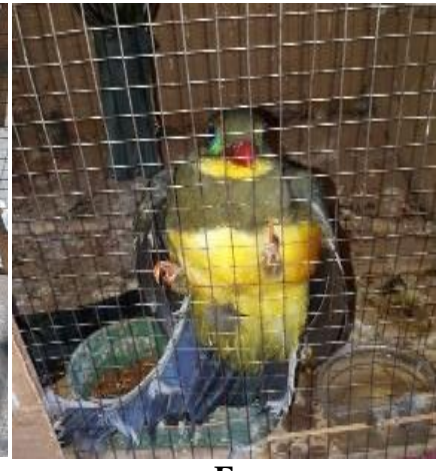

$\mathbf{F}$

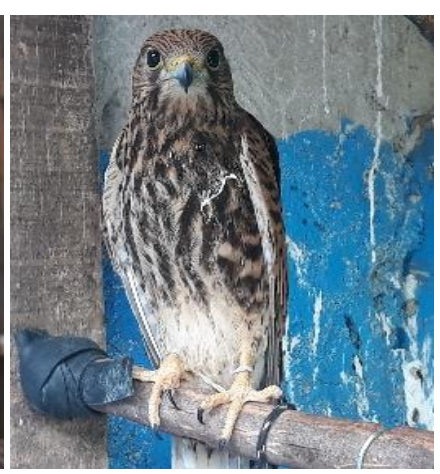

C

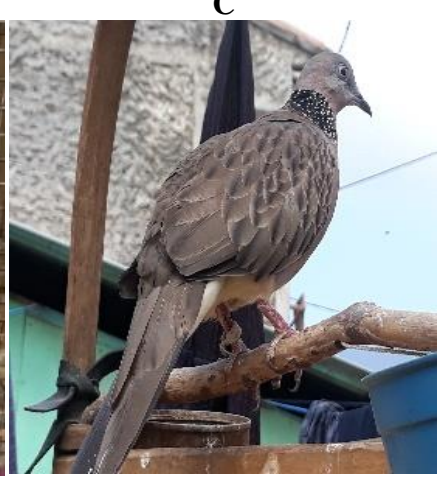

G

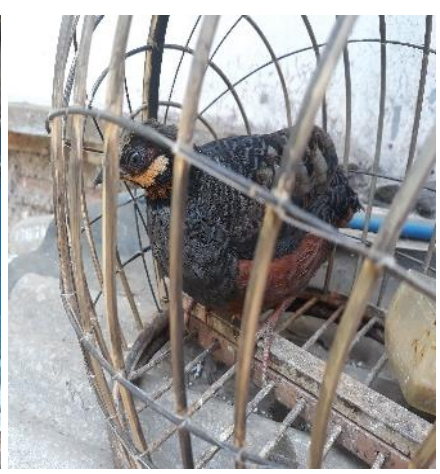

D

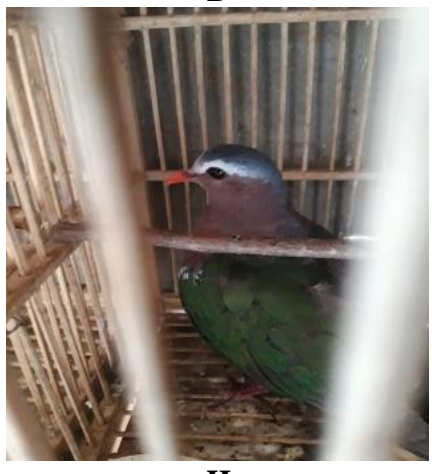

$\mathbf{H}$

Figure 4. Birds in Kertasari of upper Citarum watershed, Bandung, West Java. A. Bueuk (Ottus lempiji); B. Anis hejo (Cyornis unicolor); C. Dadali (Falco moluccensis); D. Puyuh gong-gong (Arborophila javanica); E. Koréak (Tyto alba); F. Siér/luntur gunung (Harpactes reinwardtii); G. Tikukur (Streptopelia chinensis); and H. Delimukan (Chalcophaps indica)

\section{Folkloric birds}

Example of folklore is narrated below. This information was collected once it is mentioned and comes up spontaneously in the course of everyday life, hinting at birds' important role as a vehicle for connecting two interconnected worlds. The songs and callings of certain bird species are thought to be mediating between the invisible world and the human world whether telling good fortune or bad omen, new birth, or incoming death. In the villagers' cosmology, the other world is not separated but accompanies human world daily. Birds symbolically mediate these worlds. They even have the power to tell about something that will happen in the future.

Sundanese, like Javanese, have various mythologies on birds. The Sundanese of Sukasari people, for example, have known about tikukur (Spilopelia chinensis Scopoli, Fam of Columbidae) that usually made sounds of tikukur..kuk.tikukur..kuk. According to villagers, if the sound " $k u k$ " is repeated twice or more, then it is believed the person who hears it will get good luck, especially about valuables. Although this belief is related to the wild tikukur, many villagers have this bird as a pet and put it in a cage. The cage is usually hung in the veranda, right near the entrance door of the house. It is believed that the bird's call may attract and bring good luck to the house owner. While Javanese have cultural belief that perkutut or kutut (Geopelia striata, Family of Columbidae) is considered as lucky-conveyor bird, as a result, this birds have been popularly kept as bird pet and traditionally entitled as
Katurangganing kutut which is inspired from experience of nurturing kutut bird and the legend of Java philosophy on the Katurangan of this bird (Sanjaya et al. 2017). Based on the ethnoornithology studies, it can be revealed that every ethnic community across culture in Indonesia, has cultural deep knowledge on birds, and birds are given various local folk names (Iskandar in prep).

Folkloric data was collected in the context of data collection sessions related to bird names and their identification or in a natural context. Information collected from the group's elicitation and importation session is marked CC, while those that come up with a daily course or natural context is marked NC. The following explanations deal with the meaning of the bird's name and its scientific name.

Tikukur (Spotted Dove, Spilornis chinensis Scopoli, Fam. Columbidae, Onomatopoeic) CC, NC: This bird usually makes sounds of tikukur..kuk.tikukur..kuk. According to the informants, if the sound "kuk" repeated twice or more, then it is believed the person who hears it will get good luck, especially about valuables. Although this belief is related to wild tikukur, many villagers have this bird as a pet and put it in a cage. The cage is usually hung in the veranda, right near the entrance door of the house. It is believed that bird's call may attract and bring good luck to the house owner.

Walét (Swift-Unidentified, generic name for Fam. Apodidae, Ergonymic) CC: People believe that if this bird 
enters and flies around inside the house, then luck will come to the owner of the house.

Piit (Javan munia, Lonchura leucogastroides, Fam. Estrildidae, Onomatopoeic) CC, NC): If the bird comes to a house and sings on the right side of the veranda, it is thought that a special and important guest will be coming and s/he will bring with them benefit to the house owner. Conversely, if it perches on the left side of the veranda, the guests will harm him/her.

Cangéhgar (Green jungle fowl, Gallus varius, Fam. Phsianidae, Morphonymic, literally means 'bright or shiny' ) $\mathrm{CC}$ : this bird is believed to bring good fortune to those who own it. If the rooster crowed in the middle of the night for more than two nights, it was reported that there would be pregnancy on an unmarried girl.

Bueuk (Sunda scops owl, Otus lempiji Horsfield, Fam. Strigidae, Onomatopoeic) CC, NC: It is said that the presence of this bird brings sadness. The sources of sadness could be varied, such as the death of a community member, crop failure, and others. Still, some people believe that this bird gives a hint that there will be an out of wedlock pregnancy which leads to misfortune to the community.

Gagak (Slender-billed crow, Corvus enca Horsfield, Fam. Corvidae, Onomatopoeic) CC, NC: The presence of this bird in the settlement is a sign of death, i.e. a member of the community will die. If this bird flies in a circle over someone's house, then one of the residents of the house will die shortly after.

Koréak (The Barn owl, Tyto alba Scopoli, Fam. Strigidae, Onomatopoeic) CC, NC: This bird is considered as a bird that brings disease, bad luck, bad omen, evil spirit, or even death to the community. This bird is called manukjurig [bird of ghost]. Its singing also indicates that its perching place is inhabited by a haunting ghost and to avoid or to tame it must be done by asking permission to the ghost so that we can pass it safely.

Uncuing/siit-uncuing (Rusty-breasted cuckoo, Cacomantis sepulcralis Vigor \& Horsfield, Fam. Cuculidae, Onomatopoeic) CC, NC: It is said that if a newly-wed girl died just before her first night she will become a ghost. At night, the ghost in the form of this bird flies and makes a call with the sounds like [tit...tit.....tiit], for the Sundanese, this sound means penis [titit]. Villagers believe that this bird's call signals that there will be a death of man (referring to "penis") or disaster in the community. This bird is also believed to cause havoc for people.

Korés (Brown (Grey)-checked bulbul, Alophoixus bres Lesson, Fam. Pycnonotidae, Ergonymic) CC, NC: In Sundanese 'korés' means voracious or gluttonous. It is said that there was a poor hungry young man, and when he was given food and ate it flurry, he suddenly disappeared and changed into a bird as he was about to finish eating the food. Moreover, the term 'beuteung koréseunn' referred to a man who eats food in flurry and frenzy way.

It is generally accepted that based on data presented above, like other small ethnic societies around the world, Sundanese people of Kertasari, West Java, have local knowledge on folklore in relation with birds that are inherited from their ancestors and/or obtained from their experiences in their life and their close interaction with the local environment or local ecosystem (Kane 2015; Pam 2017; Wyndham and Park 2018).

On the basis of our study, it can be concluded that it was recorded 222 bird species, representing 170 vernacular names, in which 93 of them were recorded by Koningsberger (1901-1909). Various birds of Kertasari, West Java have vernacular names that are culturally based on phononym, morphony, ergonym, and phagonym. The mountains rural people of Kertasari, West Java have local knowledge on vernacular or folk name, taxonomic, and folklore that are inherited from their ancestors by oral transmission in their mother language. Therefore, it can be inferred that there is significant role of birds for ecological, socioeconomic, and cultural functions for the Sundanese people.

\section{ACKNOWLEDGEMENTS}

This research is funded by Padjadjaran University Internal Research Grant under the scheme of 'Riset Kompetensi Dosen Unpad (RKDU)'. We are grateful to Dr. Budi Rajab who generously includes and financially supported this research in his RKDU team.

\section{REFERENCES}

Alves RRN. 2012. Relationships between fauna and people and the role of ethnozoology in animal conservation. Ethnobiol Conserv 1 (2):1-69.

Alves ASA, dos Santos L, Junior WAF, Albuquerque UP. 2016. "How and why should people classify natural resources?", in Albuquerque UP, Alves RRN. (eds.) Introduction to Ethnobiology. Springer, Heidelberg.

Agnihotri S, Si A. 2012. Solega ethnoornitology. J Ethnobiol 32 (2): 185 211.

Anderson E. 1996. Spoken Sundanese: a course in the regional language of West Java. Grasindo, Jakarta.

Berlin B. 1992. Ethnobiological classification: principles of categorization of plants and animals in traditional societies. Princeton Univ. Press, New Jersey.

Berkes F. 2008. Sacred Ecology. $2^{\text {nd }}$ ed. Routledge, London.

Bezerra DM, de Aauzo HF, Alves AGC. 2013. Birds and people in semiarid northeastern Brazil: Symbolic and medicinal relationships. J Ethnobiol Ethnomed 9: 3. DOI: 10.1186/1746-4269-9-3.

Breman J. 2015. Mobilizing labor for the global coffee market: profits from an unfree work regime in colonial Java. Amsterdam Univ. Press, Amsterdam.

Deikumah JP, Konadu VA, Kwafo R. 2015. Bird naming systems by Akan people in Ghana follow scientific nomenclature with potentials for conservation monitoring. J Ethnomed 11 (75): 1-13. DOI: 10.1186/s 13002-015-0062.

Dove MR. 1993. Uncertainty, humility, and adaptation in the tropical forest: The agricultural augury of Kantu. Ethnology 32 (2): 145-167.

Ellen R. 1993. The cultural relations of classification: an analysis of Nuaulu animal categories from Central Seram. Cambridge University Press, Cambridge.

Forth G. 2000. Eastern Sumbanese bird classification. J Ethnobiol 20: 161-192.

Geertz C. 1963. Agricultural involution: the processes of ecological exchange in Indonesia. Univ. California Press, Berkeley.

Hakim L, Abdoellah OS, Parikesit, Withaningsih S. 2020. Impact of agricultural crop type and hunting on bird communities of two villages in Bandung, West Java, Indonesia. Biodiversitas 21 (1): 5766.

Hoogerwerf 1948. Distribution of birds in Java. Treubia 19: 116-127. 
Hoogerwerf 1949a. The avifauna of Tjibodas and surroundings: including nature monument Tjibodas-Gn. Gede. The Royal Botanic Garden of Indonesia, Boitenzorg, Java. [Dutch]

Hoogerwerf $1949^{\mathrm{b}}$. The avifauna of the botanical garden in Buitenzorg (Java). the could. Botanic Garden of Indonesia, Buitenzorg, Java. [Dutch]

Hoppen Reis JHT, Van Leith B. 2016. A New Receive for Ecological Conservation in West Java. Mixing Historical Ingredients: Land Use Maps and Bird Observations. [Thesis] Radboud University, Nijmegen.

Hull K, Fergus R. 2017. Birds as Seers: An Ethno-Ornithological Approach to Omens and Prognostication Among the Ch'Orti' Maya of Guatemala. J Ethnobiol 37(4):604-620.

Iskandar J. 2012. Responses to environmental stress in the Baduy Swidden System, South Banten, Java. In Ellen R. (ed), Modern crise and traditional strategies: local ecological knowledge in Island Southeast Asia. Berghahn Books, New York.

Iskandar J. 2017. Ornithology and Ethnoornithology. Plantaxia, Yogyakarta [Indonesian].

Iskandar J. 2018. Ethnobiology, Ethnoecology and Sustainable Development. Plantaxia, Yogyakarta. [Indonesian]

Iskandar J, Iskandar BS. 2011. Sundanese agroecosystem. PT Kiblat Utama, Bandung. [Indonesian]

Iskandar J, Iskandar BS, Partasasmita R. 2016. The local knowledge of the rural people on species, role, and hunting of birds: case study in Karangwangi Village, West Java, Indonesia. Biodiversitas 17 (2): 435-446.

Iskandar J, Iskandar BS, Mulyanto D, Alfian RL, Partasasmita R. 2020. Traditional ecologocal knowledge of the bird traders on bird species, bird naming, and bird market chain: a case study in bird market Pasty Yogyakarta, Indonesia. Biodiversitas 21 (6): 2586-2602.

Kane S. 2015. Bird names and folklore from the Emberá (Chocó) in Darién, Panamá. Ethnobiol Lett 6: 32-62. DOI: 10.14237/ebl.6.1.2015.226.

Koningsberger JC. 1901-1909. The birds of Java and their economic significance. Two volumes. G. Kolff \& Co., Batavia. [Ducth]

MacKinnon JR. 1988. Field guide to the birds of Java and Bali. Gadjah Mada University Press, Yogyakarta.

Minahan JB. 2012. Ethnic groups of South Asia and the Pacific: an encyclopedia. ABC-CLIO, Santa Barbara, CA.

Müller-Gotama F. 2001. "Sundanese." Languages of the World/Materials, 369. Lincom, Munich, Munchen.
Mulyanto D, Iskandar J, Gunawan R, Partasasmita R. 2019. Ethnoornithology: identification of bird names mentioned in Kakawin Ramayana, a $9^{\text {th }}$-century Javanese poem (Java, Indonesia). Biodiversitas 20 (11): 3213-3222.

Pam GAB. 2017. Ethno-ornithology and Conservation: Traditional Ecological Knowledge (TEK) of Birds Among The Mushere and Conservation of the Dulu Forest in Mushere, Plateau State, Nigeria. [Dissertation]. University of Oxford, United Kingdom.

Nastiti TS. 2006. Old Sundanese community. In: Simanjuntak T, Hisyam M, Prasetyo B, Nastiti TS (eds.) Archaeology: Indonesian Perspective. LIPI, Jakarta.

Newing H, Eagle CM, Puri RK, Watson CW. 2011. Conducting research in conservation: a social science perspective. Routledge, London.

Roldlan-Clara B, Lopez-Medellin X, Espejel I, Arellano.2014. Literature review the use of birds as pets in Latin-America, with a detail perspective on Mexico. Ethnobiol Conserv 3 (5): 1-18. DOI: 10.15451/ec 2014-10-3.5-1-18.

Sanjaya AL, Purwantoro A, Wahyuningsih N. 2017. Katurangganing kutut. J Expresi Seni 19 (2): 174-192. [Indonesian]

Sinclair JR, Tuke L, Opiang M. 2010. 'What the locals know: comparing traditional and scientific knowledge of megapodes in Melanesia', in Tideman S, Gosler A. (eds.) Ethno-ornithology: birds, indigenous peoples, culture, and society. Earthscan, London.

Svensson T. 1991. State bureaucracy and capitalism in rural West Java: local gentry versus peasant entrepreneurs in Priangan in the $19^{\text {th }}$ and $20^{\text {th }}$ centuries. NIAS Press, Copenhagen.

Teixeira RHR, Thel TN, Ferreira JMR, de Azevedo SM, Techno Junior WR, Lyra Never RM. 2014. 2014. Local knowledge and exploitation of the avian fauna by a rural community in the semi-arid zone of Northeastern Brazil. J Ethnobiol Ethnomed 10 (81): 1-10. http://www.ethnobiomed.com/content/10/1/81.

Tidemann S, Chirgwin S, Sinclair JR. 2010. 'Indigenous knowledge, birds that have 'spoken' and science', in Tideman S, Gosler A. (eds.) Ethno-ornithology: birds, indigenous peoples, culture, and society. Earthscan, London.

Van Der Ploeg J. Van Weerd M. 2010. Agta bird names: an ethnoornithological survey in the Northern Sierra Madre Natural Park, Philippines. Forktail 26: 127-131.

Wyndham F, Park K, Fanshawe J. 2018. Listen carefully to the voices of the birds: a comparative review of birds as signs. J Ethnobiol 38(4):535-549. DOI: 10.2993/0278-0771-38.4.533. 